\title{
A MORPHIC APPROACH TO COMBINATORIAL GAMES: THE TRIBONACCI CASE*
}

\author{
ERIC Duchêne ${ }^{1}$ And Michel Rigo ${ }^{1}$
}

\begin{abstract}
We propose a variation of Wythoff's game on three piles of tokens, in the sense that the losing positions can be derived from the Tribonacci word instead of the Fibonacci word for the two piles game. Thanks to the corresponding exotic numeration system built on the Tribonacci sequence, deciding whether a game position is losing or not can be computed in polynomial time.
\end{abstract}

Mathematics Subject Classification. 91A46, 68R15, 68Q45.

\section{INTRODUCTION}

Wythoff's game is a well-known two-players take-away game played on two piles each having a finite number of tokens [19]. A move consists in selecting a pile and removing from it any positive number of tokens (same rule as for the game of Nim), or removing the same positive number of tokens from both piles. The convention is that the first player who is unable to move, loses the game. A winning strategy for this game can be derived from the Fibonacci word. A position $(x, y)$ of the game (i.e., a pair coding the number of tokens in the two piles) is said to be a $P$-position if the "previous" (i.e., the second) player can win whatever move his opponent shall make. The $P$-positions of Wythoff's game are exactly the pairs given by the positions of the $n$th occurrence of $a$ and the $n$th occurrence of $b$ in the Fibonacci word (see Rem. 3.3 for a proof of this statement). This observation gives an interesting link between combinatorial game theory and combinatorics on words.

Keywords and phrases. Two-player combinatorial game, combinatorics on words, numeration system, Tribonacci sequence.

* Post-doctoral position at University of Liège thanks to a "Subside fédéral pour la Recherche".

1 Institute of Mathematics, University of Liège, Grande Traverse 12 (B37), 4000 Liège,

Belgium; \{Eric.Duchene, M.Rigo\}@ulg.ac.be 
In [11], Fraenkel defines a removal game on three piles, namely the Raleigh game, which in his own terms is neither a generalization of the game of Nim nor of Wythoff's game. In [10], he presents a family of 2-pile games whose winning strategies are studied through the use of exotic numeration systems. In general most of the variants of Wythoff's game are solved in polynomial time through the use of numeration systems (see $[7,8,12]$ ). The originality of our approach to compute the set of $P$-positions relies on iterated morphisms instead of numeration systems. We present a variation of Wythoff's game in the sense that the $P$-positions can be derived from the natural generalization of the Fibonacci word to a three-letters alphabet, the so-called Tribonacci word: the $P$-positions are exactly the triples given by the positions of the $n$th occurrence of letter $a, b$ and $c$ in the Tribonacci word.

We propose a game based on three piles that we call the Tribonacci game. As we shall quickly see the rules of this game are symmetric on the three piles. Consequently instead of considering positions represented by arbitrary elements in $\mathbb{N}^{3}$ we can restrict ourselves to positions $(a, b, c)$ with $0 \leq a \leq b \leq c$. The moves are the following:

I. Any positive number of tokens from up to two piles can be removed.

II. From position $(a, b, c)$, one can remove a positive number $\alpha \leq a, \beta \leq b$, $\gamma \leq c$ of tokens from the respective piles consisting of $a, b, c$ tokens, subject to the condition:

$$
2 \max \{\alpha, \beta, \gamma\} \leq \alpha+\beta+\gamma .
$$

III. Let $\beta>2 \alpha>0$. From position $(a, b, c)$ one can remove the same number $\alpha$ of tokens from any two piles and $\beta$ tokens from the other one with the following condition. If $a^{\prime}$ (resp. $b^{\prime}, c^{\prime}$ ) denotes the number of tokens in the pile which contained $a$ (resp. $b, c$ ) tokens before the move, then the configuration

$$
a^{\prime}<c^{\prime}<b^{\prime}
$$

is not allowed.

As for Wythoff's game or for the game of Nim, the (normal) convention is that the first player who is unable to move, loses the game.

Remark 1.1. Rules I and II can be considered as "natural" extensions of Wythoff's rules on three piles. The third rule is necessary so as to have a set of $P$-positions where each positive integer appears at most once.

Observe that in rule III, changing the hypothesis $\beta>2 \alpha$ to $\beta \leq 2 \alpha$ gives a special case of rule II.

Example 1.2. To see how the rules of the Tribonacci game work, let us consider a few examples. Consider the configuration $(12,22,30)$. Applying rule I, one can for instance remove 12 tokens from the first and third piles to obtain $(0,18,22)$ after reordering. Unlike Wythoff's game, one can remove different numbers of tokens from two piles. So applying rule $\mathbf{I}$ by removing 12 tokens from the first pile and 5 tokens from the third one, we get from $(12,22,30)$ to the configuration $(0,22,25)$. 
Applying rule II, we can go from $(12,22,30)$ to $(1,2,3)$ because $11+20+27=$ $58 \geq 2.27$. Finally consider applying rule III, from $(a, b, c)=(12,22,30)$ if one removes $\alpha=1$ token from the first and third piles and $\beta=12$ tokens from the second pile, one gets $a^{\prime}=11, b^{\prime}=10$ and $c^{\prime}=29$ and the allowable inequalities $b^{\prime}<a^{\prime}<c^{\prime}$ to obtain configuration $(10,11,29)$. However, removing 1 token from the first two piles and 10 tokens from the third one is not allowed because we get $a^{\prime}=11, b^{\prime}=21$ and $c^{\prime}=20$ and in this case, $a^{\prime}<c^{\prime}<b^{\prime}$. But removing 1 token from the first two piles and 9 tokens from the third one is allowed. One gets $a^{\prime}=11, b^{\prime}=21$ and $c^{\prime}=21$ with the allowable configuration $a^{\prime}<b^{\prime}=c^{\prime}$.

To describe the set of $P$-positions of our Tribonacci game, we shall consider three formalisms (morphic formalism, recursive definition and game formalism) and show that they are all equivalent. Since the morphic formalism is the simplest one to produce an infinite word and to define a corresponding set of positions, we shall start with this description in Section 2. Using classical construction, the morphic description leads us to the introduction of the Tribonacci number system. In Section 3, we propose a recursive definition of the Tribonacci word. The reader will have to wait until Section 4 to see the link between the morphism and the Tribonacci game defined above. In Section 5, representations in the Tribonacci number system of the $P$-positions are shown to have a simple syntactic property depending only on their last two (least significant) digits. Moreover this allows us to have a winning strategy that can be computed in polynomial time (which is for instance a central question in [10]).

Remark 1.3. Let us mention that our game has no link with the Ducci's fournumber game problem which occurs when iterating the operator $D$ acting on 4-tuples,

$$
D(w, x, y, z):=(|w-x|,|x-y|,|y-z|,|z-w|),
$$

and which is also related in some sense to the Tribonacci sequence (for details, see for instance [18]).

\section{The MORPHIC FORMALISM}

In this section, we do not yet consider our Tribonacci game rather we simply introduce the Tribonacci sequence over the alphabet $\{a, b, c\}$ and present some of its properties which are immediate consequences of the construction using iterated morphism.

We define a sequence $\left(A_{n}, B_{n}, C_{n}\right)_{n \geq 0}$ of triples of integers directly related to the Tribonacci word. Consider the morphism $\tau:\{a, b, c\} \rightarrow\{a, b, c\}^{*}$ defined by $\tau(a)=a b, \tau(b)=a c$ and $\tau(c)=a$. Iterating this morphism starting with $a$ gives the well-known Tribonacci word $\mathbf{t}=\left(t_{n}\right)_{n \geq 1}=\lim _{n \rightarrow+\infty} \tau^{n}(a)$,

$$
\mathbf{t}=\text { abacabaabacababacabaabacabacabaabacababacabaabacabaab } \cdots .
$$

Observe that we use the convention that the first letter of $\mathbf{t}$ has index 1. Let us recall that this word appears also in symbolic dynamics or in fractal geometry (see 
TABLE 1. First values of the sequences $\left(A_{n}\right)_{n \geq 0},\left(B_{n}\right)_{n \geq 0}$ and $\left(C_{n}\right)_{n \geq 0}$.

\begin{tabular}{c|cccccccccccccccc}
\hline$n$ & 0 & 1 & 2 & 3 & 4 & 5 & 6 & 7 & 8 & 9 & 10 & 11 & 12 & 13 & 14 & 15 \\
\hline $\mathbf{A}_{\mathbf{n}}$ & 0 & 1 & 3 & 5 & 7 & 8 & 10 & 12 & 14 & 16 & 18 & 20 & 21 & 23 & 25 & 27 \\
$\mathbf{B}_{\mathbf{n}}$ & 0 & 2 & 6 & 9 & 13 & 15 & 19 & 22 & 26 & 30 & 33 & 37 & 39 & 43 & 46 & 50 \\
$\mathbf{C}_{\mathbf{n}}$ & 0 & 4 & 11 & 17 & 24 & 28 & 35 & 41 & 48 & 55 & 61 & 68 & 72 & 79 & 85 & 92 \\
\hline
\end{tabular}

for instance [6,14]). Many combinatorial properties of the Tribonacci word are studied in [17]. For $X=A, B, C$ (resp. $x=a, b, c)$, define the sets

$$
X=\left\{X_{1}<X_{2}<X_{3}<\cdots\right\}=\left\{n \in \mathbb{N} \mid t_{n}=x\right\} .
$$

Moreover we set $A_{0}=B_{0}=C_{0}=0$. The sequence $\left(A_{n}\right)_{n \geq 1}\left(\right.$ resp. $\left(B_{n}\right)_{n \geq 1}$, $\left.\left(C_{n}\right)_{n \geq 1}\right)$ is indexed as A003144 (resp. A003145, A003146) in the Sloane's encyclopedia of integer sequences [16]. These three sequences together first appeared in [4] where Table 1 is defined recursively. Many properties of these sequences were already studied in [4]. The relationship with the Tribonacci sequence was explicitly presented later in [1].

Trivially, the sets $A, B$ and $C$ give a partition of $\mathbb{N}_{\geq 1}$.

Remark 2.1. Since for any letter $x \in\{a, b, c\}, \tau(x)$ begins with $a$, it is obvious that $\Delta_{n}(a):=A_{n+1}-A_{n}$ is given by $\psi_{a}\left(t_{n}\right)$ where $\psi_{a}: a, b \mapsto 2, c \mapsto 1$.

Remark 2.2. Looking at $\tau^{2}(a)=a b a c, \tau^{2}(b)=a b a$ and $\tau^{2}(c)=a b$, one can see that $b$ always occurs in second position. Since $\tau^{2}(\mathbf{t})=\mathbf{t}$, we get that $\Delta_{n}(b):=$ $B_{n+1}-B_{n}$ is given by $\psi_{b}\left(t_{n}\right)$ where $\psi_{b}: a \mapsto 4, b \mapsto 3, c \mapsto 2$.

Remark 2.3. In the same way, looking at $\tau^{3}(a)=a b a c a b a, \tau^{3}(b)=a b a c a b$ and $\tau^{3}(c)=a b a c$, one can see that $c$ always occurs in the fourth position. Since $\tau^{3}(\mathbf{t})=\mathbf{t}$, we get that $\Delta_{n}(c):=C_{n+1}-C_{n}$ is given by $\psi_{c}\left(t_{n}\right)$ where $\psi_{c}: a \mapsto$ $7, b \mapsto 6, c \mapsto 4$.

In view of the last three remarks, we have the following result.

Theorem 2.4. With the above notation, for all $n \geq 1$, we have

$$
\left(\Delta_{n}(a), \Delta_{n}(b), \Delta_{n}(c)\right) \in\{(2,4,7),(2,3,6),(1,2,4)\} .
$$

In particular, $\Delta_{n}(a)+\Delta_{n}(b)+1=\Delta_{n}(c)$.

The Tribonacci number system is a positional numeration system given by the linearly recurrent sequence $T_{0}=1, T_{1}=2, T_{2}=4$ and $T_{k+3}=T_{k+2}+T_{k+1}+T_{k}$ for all $k \geq 0$. Using a greedy algorithm [9] and a variation of the Zeckendorf's expansion defined for the Fibonacci sequence [20], any positive integer $n$ has a unique decomposition

$$
n=\sum_{i=0}^{\ell} c_{i} T_{i}
$$


TABLE 2. The Tribonacci representations of the first 20 positive integers.

\begin{tabular}{rr|rr|cc|cc|cc}
\hline 1 & 1 & 5 & 101 & 9 & 1010 & 13 & 10000 & 17 & 10100 \\
2 & 10 & 6 & 110 & 10 & 1011 & 14 & 10001 & 18 & 10101 \\
3 & 11 & 7 & 1000 & 11 & 1100 & 15 & 10010 & 19 & 10110 \\
4 & 100 & 8 & 1001 & 12 & 1101 & 16 & 10011 & 20 & 11000 \\
\hline
\end{tabular}

TABLE 3. First words in $M$ and their numerical value.

\begin{tabular}{c|cccccc}
\hline$n$ & 1 & 2 & 3 & 4 & 5 & $\cdots$ \\
\hline$M_{n}$ & $\varepsilon$ & 1 & 10 & 11 & 100 & $\cdots$ \\
$\operatorname{val}\left(M_{n}\right)$ & 0 & 1 & 2 & 3 & 4 & $\cdots$ \\
\hline
\end{tabular}

with $c_{i} \in\{0,1\}$ for all $i \in\{0, \ldots, \ell-1\}, c_{\ell}=1$ and no three consecutive digits being equal to 1 . This result is a consequence of the following facts:

- For all $n \geq 1$, we have $T_{n} / T_{n-1} \leq 2$. Therefore all the coefficients $c_{i}$ appearing in the decomposition belong to $\{0,1\}$. Indeed, assume that there exists $i \in\{0, \ldots, \ell\}$ such that $c_{i} \geq 2$. Then $c_{i} T_{i}+\cdots+c_{0} T_{0} \geq T_{i+1}$ which contradicts the greediness of the algorithm.

- Again by greediness of the algorithm and by definition of the Tribonacci sequence, no three consecutive digits can be equal to 1 .

- Uniqueness of the decomposition follows from the fact that any decomposition of $n=\sum_{i=0}^{\ell} c_{i} T_{i}$ with $c_{i} \in\{0,1\}$ for all $i \in\{0, \ldots, \ell-1\}$, $c_{\ell}=1$ and no three consecutive digits being equal to 1 is a greedy decomposition. We proceed by induction. We have $c_{0} T_{0}<T_{1}$. Assume that $c_{i} T_{i}+\cdots+c_{0} T_{0}<T_{i+1}$ for $0 \leq i<\ell$. Let $D=c_{i+1} T_{i+1}+\cdots+c_{0} T_{0}$. If $c_{i+1}=0$ then $D<T_{i+1}<T_{i+2}$. If $c_{i+1}=1$ then $D<2 T_{i+1} \leq T_{i+2}$.

The word $\rho_{T}(n)=c_{\ell} \cdots c_{0}$ is said to be the Tribonacci representation of $n$. It is convenient to assume that 0 can be equally represented by the word 0 or by the empty word $\varepsilon$.

Remark 2.5. Using a result linking morphic sequences and (abstract) numeration systems ([15], Th. 24), it is not difficult to see that $t_{n}=a\left(\right.$ resp. $\left.t_{n}=b, t_{n}=c\right)$ if and only if the Tribonacci representation of $n-1$ ends with 0 (resp. 01, 11).

Indeed, in a canonical way we can associate to the morphism $\tau$ a deterministic finite automaton $\mathcal{A}$ having $\{a, b, c\}$ as set of states and $\{0,1\}$ as alphabet (this kind of construction already appeared in the seminal paper [5]). If $\delta:\{a, b, c\} \times\{0,1\} \rightarrow$ $\{a, b, c\}$ is the transition function of $\mathcal{A}$, then

$$
\delta(a, 0)=a, \delta(a, 1)=b, \delta(b, 0)=a, \delta(b, 1)=c, \delta(c, 0)=a .
$$

Notice that $\delta$ is not a total function, i.e., $\mathcal{A}$ is not complete. Let $M=L(\mathcal{A}) \backslash$ $0\{0,1\}^{*}$ where $L(\mathcal{A})$ is the language accepted by the automaton $\mathcal{A}$ where all states 
are final and $a$ is the initial state. Let $M_{n}$ be the $n$th word $(n \geq 1)$ of the genealogically ordered language $M$. (Suppose $x, y$ are two words on a totally ordered alphabet $(\Sigma,<)$. Then $x$ is genealogically less than $y$ if $|x|<|y|$ or, if $|x|=|y|$ and $x=u a v, y=u b w$ where $u, v, w \in \Sigma^{*}, a, b \in \Sigma$ and $\left.a<b\right)$. Since $L(\mathcal{A})=\{0,1\}^{*} \backslash\{0,1\}^{*} 111\{0,1\}^{*}$, the numerical value $\operatorname{val}\left(M_{n}\right)$ of $M_{n}$ with respect to the Tribonacci system is $\operatorname{val}\left(M_{n}\right)=n-1$. See for instance Table 3 .

Moreover, using again [15], feeding $\mathcal{A}$ with $M_{n}$ leads to the state $t_{n}$.

\section{ReCURSive Definition}

Observe that the $n$th occurrence of $c$ in $\mathbf{t}$ belongs to the image under $\tau$ of the $n$th occurrence of $b$ in $\mathbf{t}$ which appears itself in the image under $\tau$ of the $n$th occurrence of $a$. Hence it is clear that for all $n \geq 1, B_{n}-A_{n}$ and $C_{n}-B_{n}$ are positive, i.e., $A_{n}<B_{n}<C_{n}$. The aim of this section is to obtain a recursive characterization of $\mathbf{t}$ (see Th. 3.1). Notice that this characterization differs from the one given initially in [4] (especially for the formula giving $B_{n}$ ). As usual, the notation Mex stands for Minimum EXcluded value [2].

Theorem 3.1. Assume $X_{n}$ denotes the position of the nth occurrence of $x$ in the Tribonacci word $\mathbf{t}, X=A, B, C$ (resp. $x=a, b, c)$. For all $n \geq 1$, t satisfies

$$
\left\{\begin{array}{l}
A_{n}=\operatorname{Mex}\left\{A_{i}, B_{i}, C_{i}: 0 \leq i<n\right\} \\
B_{n}=A_{n}+\operatorname{Mex}\left\{B_{i}-A_{i}, C_{i}-B_{i}: 0 \leq i<n\right\} \\
C_{n}=A_{n}+B_{n}+n
\end{array}\right.
$$

Moreover, $\mathbf{t}$ is the only word over a ternary alphabet $\{a, b, c\}$ satisfying these relations.

The following result is also discussed in [1] where a "simple" construction of $\mathbf{t}$ is given. For the sake of completeness and since all the necessary material is already introduced, we recall the proof.

Lemma 3.2. Assume $X_{n}$ denotes the position of the $n$th occurrence of $x$ in the Tribonacci word $\mathbf{t}, X=A, B, C$ (resp. $x=a, b, c)$. For all $n \geq 1$, we have

$$
C_{n}=A_{n}+B_{n}+n
$$

Proof. For $X=A, B, C$ (resp. $x=a, b, c)$, we use notation of Remarks 2.1, 2.2 and 2.3 , it is clear that for all $n \geq 1$,

$$
X_{n}=X_{1}+\sum_{i=1}^{n-1} \Delta_{i}(x)
$$


Therefore, using Theorem 2.4, we get

$$
C_{n}=4+\sum_{i=1}^{n-1}\left(\Delta_{i}(a)+\Delta_{i}(b)+1\right)=A_{1}+\sum_{i=1}^{n-1} \Delta_{i}(a)+B_{1}+\sum_{i=1}^{n-1} \Delta_{i}(b)+n
$$

and the result follows.

Remark 3.3 (A characterization of the Fibonacci word). Consider the morphism $\varphi: a \mapsto a b, b \mapsto a$ generating the celebrated Fibonacci word $\mathbf{f}=\lim _{n \rightarrow \infty} \varphi^{n}(a)=$ abaababaabaababaabab .. e.g., see [13]. Let us denote by $a_{n}$ (resp. $\left.b_{n}\right)$ the position of the $n$th occurrence of $a$ (resp. b) in $\mathbf{f}$, i.e.,

$$
\left(a_{n}\right)_{n \geq 1}=1,3,4,6,8,9, \ldots \quad \text { and } \quad\left(b_{n}\right)_{n \geq 1}=2,5,7,10,13,15, \ldots
$$

It is not difficult to see that, for all $n \geq 1$, the Fibonacci word is the only word over a binary alphabet satisfying

$$
a_{n}=\operatorname{Mex}\left\{a_{i}, b_{i}: 0 \leq i<n\right\} \quad \text { and } \quad b_{n}=a_{n}+n .
$$

Indeed, the $n$th occurrence of $a$ produces using $\varphi$ the $n$th occurrence of $b$ in $\mathbf{f}$. Since all $b$ 's are produced by letters $a$, during the iteration process, all the remaining gaps are filled with $a$ and $a_{n}=\operatorname{Mex}\left\{a_{i}, b_{i}: 0 \leq i<n\right\}$.

For the second part, since $a b$ is a prefix of $\mathbf{f}$ then $b_{1}=a_{1}+1$. Assume that $b_{n-1}=a_{n-1}+n-1$ for some $n \geq 2$. Notice that if $a_{n}-a_{n-1}=1$ (resp. 2$)$ then $b_{n}-b_{n-1}=2$ (resp. 3). Therefore, $b_{n}=a_{n}+n$.

The sequence $\left(a_{n}, b_{n}\right)_{n \geq 1}$ was studied in $[8,19]$ from a game theoretical point of view. In [19] it is shown that the $P$-positions of Wythoff's game are exactly the $\left(x_{n}, y_{n}\right)$ satisfying the same relation as (1). Consequently, the $P$-positions of Wythoff's game are exactly the pairs given by the positions of the $n$th occurrence of $a$ and the $n$th occurrence of $b$ in the Fibonacci word.

If $\mathbf{x}=\left(x_{n}\right)_{n \geq 0}$ is an infinite word over a finite alphabet of integers, then we denote by $S(\mathbf{x})$ the corresponding summatory sequence, i.e., for all $n \geq 0$,

$$
S(\mathbf{x})_{n}=\sum_{i=0}^{n} x_{i}
$$

Let $Y \subseteq \mathbb{N}$ be a set of integers. The characteristic sequence of $Y$ is an infinite word $\chi_{Y}=\left(x_{n}\right)_{n \geq 0}$ over $\{0,1\}$ defined by $x_{i}=1$ if and only if $i \in Y$.

Lemma 3.4. Let $\mu:\{a, b, c\} \rightarrow\{2,3\}^{*}$ and $\nu:\{a, b, c\} \rightarrow\{1,2\}^{*}$ be two morphisms defined by

$$
\mu(a)=\mu(b)=3, \mu(c)=2, \nu(a)=\nu(b)=21 \text { and } \nu(c)=2 .
$$

For any infinite word $w \in\{a, b, c\}^{\omega}$, the sets

$$
S_{\mu}=\left\{S(2 \mu(w))_{n} \mid n \geq 0\right\} \quad \text { and } \quad S_{\nu}=\left\{S(1 \nu(w))_{n} \mid n \geq 0\right\}
$$

form a partition of $\mathbb{N}_{\geq 1}$. 
Proof. Let $\chi_{\mu}$ (resp. $\chi_{\nu}$ ) be the characteristic sequence of $S_{\mu}$ (resp. $S_{\nu}$ ). Each letter of $w$ gives a subword of $\chi_{\mu}$ (resp. $\chi_{\nu}$ ), assuming that a 1 appears earlier (which is always the case since $\chi_{\mu}$ (resp. $\chi_{\nu}$ ) starts with 001 (resp. 01). We give the possible subwords written in bold case:

\begin{tabular}{c|ccc}
\hline & $a$ & $b$ & $c$ \\
\hline$\chi_{\mu}$ & 1001 & 1001 & 101 \\
$\chi_{\nu}$ & 1011 & 1011 & 101 \\
\hline
\end{tabular}

It is clear that in $\chi_{\mu}$, each 1 is preceded with 0 (the values taken by $\mu$ are at least 2 which means that $S_{\mu}$ does not contain two consecutive integers) and in $\chi_{\nu}$, each 1 is followed by 0 (the images of $\nu$ all start with 2 ). Since we consider the summatory sequences obtained from the sequences $2 \mu(w)$ and $1 \nu(w)$ starting respectively with 2 and 1 , the subwords obtained in $\chi_{\mu}$ and $\chi_{\nu}$ corresponding to the same letter in $w$ are thus shifted by one. Namely, we have:

\begin{tabular}{c|ccc}
\hline & $a$ & $b$ & $c$ \\
\hline$\chi_{\mu}$ & 01001 & 01001 & 0101 \\
$\chi_{\nu}$ & 10110 & 10110 & 1010 \\
\hline
\end{tabular}

Gluing together such possible factors gives the result.

Remark 3.5. The above result can be trivially extended to two morphisms defined on an arbitrary finite alphabet $\Gamma$ and taking values in an alphabet $\Sigma \subset \mathbb{N}_{\geq 1}$ of integers. Suppose that for all $a \in \Gamma, \mu(a)$ does not start nor end with 1 and is of the form

$$
\mu(a)=w_{1} 1 a_{2} w_{2} 1 a_{3} w_{3} \cdots 1 a_{k} w_{k} \quad \text { where } a_{i} \in \Sigma \backslash\{1\}, w_{i} \in \Sigma^{*} .
$$

It is obvious that if for all $n \geq 2$, we set $\zeta(n):=2(1)^{n-2}$, then the morphism $\nu$ defined by

$$
\nu(a)=\zeta\left(w_{1}\right) 3(1)^{a_{2}-2} \zeta\left(w_{2}\right) 3(1)^{a_{3}-2} \zeta\left(w_{3}\right) \cdots 3(1)^{a_{k}-2} \zeta\left(w_{k}\right)
$$

can be used to define a partition of $\mathbb{N}_{\geq 1}$.

Corollary 3.6. Assume $X_{n}$ denotes the position of the nth occurrence of $x$ in the Tribonacci word $\mathbf{t}, X=A, B, C$ (resp. $x=a, b, c)$. The sets

$$
\left\{B_{i}-A_{i} \mid i \geq 1\right\}=\{1,3,4,6,7,9,10,12,14,15,17,18,20,21,23,24, \ldots\}
$$

and

$$
\left\{C_{i}-B_{i} \mid i \geq 1\right\}=\{2,5,8,11,13,16,19,22,25,28,31,33,36,39,42, \ldots\}
$$

give a partition of $\mathbb{N}_{\geq 1}$. In particular, the mappings $n \mapsto B_{n}-A_{n}$ and $n \mapsto C_{n}-B_{n}$ are increasing. 
Proof. In view of Theorem 2.4, the sequence $\left(\left(C_{n+1}-B_{n+1}\right)-\left(C_{n}-B_{n}\right)\right)_{n \geq 1}=$ $\left(\Delta_{n}(c)-\Delta_{n}(b)\right)_{n>1}$ is given by $\mu(\mathbf{t})$ where $\mu$ is the same as in the previous lemma (indeed, for all $x \in\{a, b, c\}, \mu(x)=\psi_{c}(x)-\psi_{b}(x)$ ). Moreover, $C_{1}-B_{1}=2$. Therefore, the set $\left\{C_{i}-B_{i} \mid i \geq 1\right\}$ is given by the summatory sequence $S(2 \mu(\mathbf{t}))$.

In the same way, the sequence $\left(\left(B_{n+1}-A_{n+1}\right)-\left(B_{n}-A_{n}\right)\right)_{n \geq 1}=\left(\Delta_{n}(b)-\right.$ $\left.\Delta_{n}(a)\right)_{n \geq 1}$ is given by $\gamma(\mathbf{t})$ where $\gamma(a)=2, \gamma(b)=\gamma(c)=1$. Notice that

$$
\gamma \circ \tau=\nu
$$

where $\nu$ is the same as in the previous lemma. Since $B_{1}-A_{1}=1$, the set $\left\{B_{i}-A_{i} \mid i \geq 1\right\}$ is given by $S(1 \gamma(\mathbf{t}))$. To conclude, recall that $\mathbf{t}=\tau(\mathbf{t})$. Therefore,

$$
S(1 \gamma(\mathbf{t}))=S(1 \gamma(\tau(\mathbf{t})))=S(1 \nu(\mathbf{t}))
$$

and the result follows using the previous lemma.

With the following lemma, we get the proof of Theorem 3.1.

Lemma 3.7. Assume $X_{n}$ denotes the position of the $n$th occurrence of $x$ in the Tribonacci word $\mathbf{t}, X=A, B, C$ (resp. $x=a, b, c)$. For all $n \geq 1$, we have

$$
\left\{\begin{array}{l}
A_{n}=\operatorname{Mex}\left\{A_{i}, B_{i}, C_{i}: 0 \leq i<n\right\} \\
B_{n}=A_{n}+\operatorname{Mex}\left\{B_{i}-A_{i}, C_{i}-B_{i}: 0 \leq i<n\right\} .
\end{array}\right.
$$

Proof. We proceed by induction on $n$. Assume that the result holds for all values less than $n \geq 2$. Let $t:=B_{n-1}-A_{n-1}=\operatorname{Mex}\left\{B_{i}-A_{i}, C_{i}-B_{i}: 0 \leq i<n-1\right\}$. Then as shown in the proof of the previous corollary, $B_{n}-A_{n}=t+1$ or $t+2$ (the images of $\gamma$ are 1 or 2). In the first case, again using Corollary 3.6, if $B_{n}-A_{n}=t+1$ then $C_{n-1}-B_{n-1} \neq t+1$ and we conclude that $B_{n}-A_{n}=t+1=\operatorname{Mex}\left\{B_{i}-A_{i}, C_{i}-\right.$ $\left.B_{i}: 0 \leq i<n\right\}$. In the second case, assume that $B_{n}-A_{n}=t+2$. Therefore, we just have to show that there exists $m<n$ such that $C_{m}-B_{m}=t+1$. The existence of such a $m>0$ is again guaranteed by Corollary 3.6 and $m<n$ because for all $x=a, b, c, \mu(x)-\gamma(x) \geq 1$. Indeed, we have $C_{i}-A_{i} \geq i$ for all $i \geq 1$ and thus since $B_{n-1}-A_{n-1}=t, C_{n-1}-B_{n-1} \geq t+n-1 \geq t+1$ for $n \geq 2$. Moreover $i \mapsto C_{i}-B_{i}$ is increasing hence $m<n$.

Up to now, and in view of Lemma 3.2, we have shown that positions of the $n$th occurrences of $b$ and $c$ in $\mathbf{t}$ are well-defined and appear after the $n$th occurrence of $a$. To conclude the proof, observe that filling the gaps with $a$ 's corresponds exactly to the Mex definition.

Let $X=A, B, C$. In what follows, to avoid many intricated subscripts, we equally consider sequences $\left(X_{n}\right)_{n \geq 0}$ as functions $X: \mathbb{N} \rightarrow \mathbb{N}: n \mapsto A_{n}$. We can therefore use composition of functions. The following two lemmas give properties of $\mathbf{t}$ that we use in the next section.

Lemma 3.8. Assume $X_{n}$ denotes the position of the nth occurrence of $x$ in the Tribonacci word $\mathbf{t}, X=A, B, C$ (resp. $x=a, b, c)$. We have for all $n \geq 1$,

$$
C(n)-B(n)=1+B(A(n))-A(A(n))
$$


and for all $n \geq 0$,

$$
C(n)-B(n)+1=B(A(n)+1)-A(A(n)+1) .
$$

Proof. If $y=y_{1} \cdots y_{k}$ is a finite word over a finite alphabet of integers, similar to the infinite case we use the notation $S$ to denote $S(y)=y_{1}+\cdots+y_{k}$. Let $\mathbf{t}[1, n]:=t_{1} \cdots t_{n}$ be the prefix of length $n$ of $\mathbf{t}$. Notice that

$$
|\tau(\mathbf{t}[1, n])|=A(n+1)-1, \text { i.e., } \tau(\mathbf{t}[1, n])=\mathbf{t}[1, A(n+1)-1] .
$$

Indeed, for all $i=1, \ldots, n, \tau\left(t_{i}\right)$ contains exactly one $a$. Therefore, to determine the length of $\tau(\mathbf{t}[1, n])$, one only needs to look for the $(n+1)$ st $a$ in $\mathbf{t}$.

It is clear that for all $n \geq 1, C(n)-B(n)$ is given by $S(2 \mu(\mathbf{t}[1, n-1]))$ and $B(n)-A(n)=S(1 \gamma(\mathbf{t}[1, n-1]))$. We obtain, for all $n \geq 1$

$$
\begin{aligned}
C(n)-B(n) & =S(2 \mu(\mathbf{t}[1, n-1]))=S(2 \nu(\mathbf{t}[1, n-1])) \\
& =S(2 \gamma(\tau(\mathbf{t}[1, n-1])))=S(2 \gamma(\mathbf{t}[1, A(n)-1])) \\
& =1+S(1 \gamma(\mathbf{t}[1, A(n)-1]))=1+B(A(n))-A(A(n)) .
\end{aligned}
$$

To get the second formula, notice that

$$
B(A(n)+1)-A(A(n)+1)-[B(A(n))-A(A(n))]=\gamma\left(t_{A(n)}\right)=\gamma(a)=2 .
$$

Lemma 3.9. Assume $X_{n}$ denotes the position of the nth occurrence of $x$ in the Tribonacci word $\mathbf{t}, X=A, B, C$ (resp. $x=a, b, c)$. For all $n \geq 0$, we have

$$
B(n)=A(A(n)+1)-1 .
$$

Proof. From (2), we get $\tau(\mathbf{t}[1, A(n)])=\mathbf{t}[1, A(A(n)+1)-1]$ and since $t_{A(n)}$ is the $n$th occurrence of $a$ in $\mathbf{t}$, then the last letter of $\tau(\mathbf{t}[1, A(n)])$ is the $n$th occurrence of $b$ in $\mathbf{t}$.

\section{GAME FORMALISM}

We have collected in the previous section all the necessary results that we need to prove our main result about the Tribonacci game.

Theorem 4.1. The set $\mathcal{S}=\left\{\left(A_{n}, B_{n}, C_{n}\right) \mid n \geq 0\right\}$ is the set of P-positions of the Tribonacci game.

Proof. We proceed in two steps (in graph theoretical terms, we show that the set of $P$-positions is the kernel of the acyclic ${ }^{1}$ game graph $G$ whose set of vertices is the set of positions and where a directed edge from $u$ to $v$ exists iff there is a legal move between the positions corresponding to $u$ and $v$ ).

\footnotetext{
${ }^{1}$ Since tokens are taken from the piles at each move, it is obvious that the game graph of the Tribonacci game is acyclic. This ensures existence and uniqueness of the kernel.
} 
- Whatever the chosen rule (I, II or III) is, a player moving from a position $\left(A_{n}, B_{n}, C_{n}\right)$ in $\mathcal{S}$ always lands in a position not in $\mathcal{S}$.

- Given a position $(a, b, c)$ not in $\mathcal{S}$, there exists a move to some position $\left(A_{n}, B_{n}, C_{n}\right) \in \mathcal{S}$.

Let us first introduce some notation to simplify our presentation. Remember that due to the symmetry of the rules, we have only to consider positions $(a, b, c) \in \mathbb{N}^{3}$ such that $a \leq b \leq c$. So the notation $(a, b, c)$ contains the extra information $a \leq b \leq c$. If one removes non-negative integers $\alpha, \beta$ and $\gamma$ respectively from the three piles $a, b$ and $c$, we get $a^{\prime}=a-\alpha, b^{\prime}=b-\beta$ and $c^{\prime}=c-\gamma$ and we use brackets $\left[a^{\prime}, b^{\prime}, c^{\prime}\right]$ instead of parentheses to specify that the resulting triple is not necessarily ordered. We shall therefore write

$$
(a, b, c) \stackrel{\alpha, \beta, \gamma}{\longrightarrow}\left[a^{\prime}, b^{\prime}, c^{\prime}\right]
$$

Notice that if $a^{\prime}<b^{\prime}=c^{\prime}$ or $a^{\prime}=b^{\prime}<c^{\prime}$ or $a^{\prime}=b^{\prime}=c^{\prime}$, we consider naturally that the triple remains ordered. In particular, we write $\left[a^{\prime}, b^{\prime}, c^{\prime}\right]=\left(a^{\prime}, b^{\prime}, c^{\prime}\right)$ if the order of the piles is unchanged after the move. Finally, we write

$$
(a, b, c) \stackrel{\mathbf{R}}{\longrightarrow}(x, y, z)
$$

to specify that the position $(x, y, z)$ has been obtained from $(a, b, c)$ using rule $\mathbf{R}$ (I, II or III). As an example, we write

$$
(12,22,30) \stackrel{(11,0,12)}{\longrightarrow}[1,22,18] \neq(1,18,22) \quad \text { and } \quad(12,22,30) \stackrel{\mathbf{I}}{\longrightarrow}(1,18,22) .
$$

- For the first part of the proof, we show that $\mathcal{S}$ is a stable subset of the game graph $G$, i.e., $\mathcal{S}$ is a set of pairwise non-adjacent vertices. Assume that a player moves from a position $\left(A_{n}, B_{n}, C_{n}\right)$ in $\mathcal{S}$ to another position $\left(A_{m}, B_{m}, C_{m}\right)$ in $\mathcal{S}$. Clearly $m<n$. We show that such a move never occurs by considering the possible application of the different rules. First observe that we cannot apply rule $\mathbf{I}$ to obtain

$$
\left(A_{n}, B_{n}, C_{n}\right) \stackrel{\mathbf{I}}{\longrightarrow}\left(A_{m}, B_{m}, C_{m}\right)
$$

for some $m$. Since $\left\{A_{j} \mid j \geq 1\right\} \cup\left\{B_{j} \mid j \geq 1\right\} \cup\left\{C_{j} \mid j \geq 1\right\}$ is a partition of $\mathbb{N}_{>1}$, none of the elements in the first triple is equal to any element of the second triple. But with rule $\mathbf{I}$ at least one of the piles should remain unchanged which is a contradiction. Now consider applying rule II:

$$
\left(A_{n}, B_{n}, C_{n}\right) \stackrel{\mathbf{I I}}{\longrightarrow}\left(A_{m}, B_{m}, C_{m}\right) .
$$

As a first case, suppose that the resulting position has been obtained from

$$
\left(A_{n}, B_{n}, C_{n}\right) \stackrel{\alpha, \beta, \gamma}{\longrightarrow}\left[A_{m}, B_{m}, C_{m}\right]=\left(A_{m}, B_{m}, C_{m}\right)
$$


with $\alpha, \beta, \gamma>0$ satisfying the condition of rule II. Using Theorem $2.4,(\alpha, \beta, \gamma)=$ $\left(A_{n}-A_{m}, B_{n}-B_{m}, C_{n}-C_{m}\right)$ is a linear combination (with non-negative coefficients) of the vectors $(1,2,4),(2,3,6)$ and $(2,4,7)$ and therefore $\gamma>\alpha+\beta$. This contradicts the fact that rule II has been applied. As a second case, assume that the position $\left(A_{m}, B_{m}, C_{m}\right)$ has been obtained from

$$
\left(A_{n}, B_{n}, C_{n}\right) \stackrel{\alpha, \beta, \gamma}{\longrightarrow}[x, y, z] \neq\left(A_{m}, B_{m}, C_{m}\right) .
$$

Otherwise stated $[x, y, z]$ is a non-trivial permutation of $\left(A_{m}, B_{m}, C_{m}\right)$. Since for $X=A, B, C, X_{n}>X_{m}$, there exist some $\alpha^{\prime}, \beta^{\prime}, \gamma^{\prime}>0$ such that

$$
\left(A_{n}, B_{n}, C_{n}\right) \stackrel{\alpha^{\prime}, \beta^{\prime}, \gamma^{\prime}}{\longrightarrow}\left[A_{m}, B_{m}, C_{m}\right]=\left(A_{m}, B_{m}, C_{m}\right)
$$

In view of the first case, we still have

$$
\gamma^{\prime}>\alpha^{\prime}+\beta^{\prime}
$$

Moreover our key argument is to observe that

$$
\alpha+\beta+\gamma=\alpha^{\prime}+\beta^{\prime}+\gamma^{\prime}
$$

If $z=C_{m}$, then $\gamma=\gamma^{\prime}$. Otherwise $z=A_{m}$ or $B_{m}$ which are less than $C_{m}$, therefore $\gamma>\gamma^{\prime}$. Taking into account these two cases and using (3) and (4), we obtain $\gamma \geq \gamma^{\prime}>\alpha^{\prime}+\beta^{\prime} \geq \alpha+\beta$ which also contradicts the fact that rule II should have been applied. Lastly, consider applying rule III:

$$
\left(A_{n}, B_{n}, C_{n}\right) \stackrel{\text { III }}{\longrightarrow}\left(A_{m}, B_{m}, C_{m}\right)
$$

where the resulting position has been obtained from

$$
\left(A_{n}, B_{n}, C_{n}\right) \stackrel{\alpha, \beta, \gamma}{\longrightarrow}[x, y, z]
$$

We have five cases to consider depending on which permutation of $\left(A_{m}, B_{m}, C_{m}\right)$ corresponds to the reordering of $[x, y, z]$ (remember that one permutation is forbidden with rule III). With the same reasoning as before, there exist $\alpha^{\prime}, \beta^{\prime}, \gamma^{\prime}>0$ such that

$$
\left(A_{n}, B_{n}, C_{n}\right) \stackrel{\alpha^{\prime}, \beta^{\prime}, \gamma^{\prime}}{\longrightarrow}\left[A_{m}, B_{m}, C_{m}\right]=\left(A_{m}, B_{m}, C_{m}\right)
$$

Clearly (3) and (4) still hold and invoking again Theorem 2.4, we can conclude that

$$
\alpha^{\prime}<\beta^{\prime}<\gamma^{\prime}
$$

(a) If $[x, y, z]=\left(A_{m}, B_{m}, C_{m}\right)$, then with the same reasoning $\alpha<\beta<\gamma$. But since we have applied rule III (we have to remove the same number of tokens from two piles), we should have $\alpha=\beta, \alpha=\gamma$ or $\beta=\gamma$ leading to a contradiction. 
(b) If $[x, y, z]=\left[C_{m}, B_{m}, A_{m}\right]$, then $\beta^{\prime}=\beta, \alpha<\alpha^{\prime}$ and $\gamma^{\prime}<\gamma$. Consequently, $\alpha<\alpha^{\prime}<\beta^{\prime}<\gamma^{\prime}<\gamma$ and $\alpha<\beta<\gamma$ contradicts rule III.

(c) If $[x, y, z]=\left[B_{m}, A_{m}, C_{m}\right]$, then $\gamma^{\prime}=\gamma, \alpha^{\prime}>\alpha$ and $\beta^{\prime}<\beta$. If $\alpha=\beta$, then $A_{n}-B_{m}=B_{n}-A_{m}$ and we get $B_{n}-A_{n}=A_{m}-B_{m}$. This is a contradiction because $B_{j}-A_{j}>0$ for $j>0$ and thus $B_{n}-A_{n}>0$ but $A_{m}-B_{m} \leq 0$. If $\alpha=\gamma$, then $A_{n}-B_{m}=C_{n}-C_{m}$ and we get $A_{n}-A_{m}>A_{n}-B_{m}=C_{n}-C_{m}$. This is a contradiction by Theorem 2.4. For the last case, assume that $\beta=\gamma$. Since $\gamma^{\prime}=\gamma$, using (4) we get $\alpha^{\prime}+\beta^{\prime}=\alpha+\beta$ and $\alpha^{\prime}-\alpha=\beta-\beta^{\prime}=\gamma-\beta^{\prime}=\gamma^{\prime}-\beta^{\prime}$. Consequently, $\alpha^{\prime} \geq \gamma^{\prime}-\beta^{\prime}$ which contradicts (3). We have therefore shown that $\alpha, \beta, \gamma$ are three distinct integers which contradicts rule III.

(d) If $[x, y, z]=\left[B_{m}, C_{m}, A_{m}\right]$, then $\alpha<\alpha^{\prime}, \beta<\beta^{\prime}$ and $\gamma>\gamma^{\prime}$. If $\alpha=\gamma$ (resp. $\beta=\gamma$ ), then $\gamma^{\prime}<\alpha^{\prime}$ (resp. $\gamma^{\prime}<\beta^{\prime}$ ) which contradicts (5). Let us now assume that $\alpha=\beta$, then $A_{n}-B_{m}=B_{n}-C_{m}$ and we conclude as in (c) using Corollary 3.6. Once again, we have shown that $\alpha, \beta, \gamma$ are three distinct integers which contradicts rule III.

(e) If $[x, y, z]=\left[C_{m}, A_{m}, B_{m}\right]$, then $\alpha<\alpha^{\prime}, \beta>\beta^{\prime}$ and $\gamma>\gamma^{\prime}$. This case can be treated as the previous one. We apply Corollary 3.6 to show that $\beta \neq \gamma$.

- For second part of the proof, we show that $\mathcal{S}$ is an absorbing subset of the game graph $G$, i.e., any vertex not in $\mathcal{S}$ has a successor in $\mathcal{S}$. Let $(a, b, c)$ be a position not in $\mathcal{S}$. There are a few cases that we can directly deal with:

- if $a=0$, then $(a, b, c) \stackrel{\mathrm{I}}{\longrightarrow}(0,0,0)=\left(A_{0}, B_{0}, C_{0}\right)$;

- if $b=c$, then $(a, b, c) \stackrel{\text { II }}{\longrightarrow}(0,0,0)$;

- if $a=b$ and $c \leq 2 a$, then $(a, b, c) \stackrel{\text { II }}{\longrightarrow}(0,0,0)$;

- if $a=b$ and $c>2 a$, then $(a, b, c) \stackrel{\text { III }}{\longrightarrow}(0,0,0)$;

- if $c \leq a+b$ then $(a, b, c) \stackrel{\text { II }}{\longrightarrow}(0,0,0)$.

So in what follows, we may assume that

$$
0<a<b<c \text { and } c>a+b \text {. }
$$

There exists a unique $n>0$ such that $a$ is a component of the triple $\left(A_{n}, B_{n}, C_{n}\right)$. We therefore discuss three cases, according to $a$.

(a) If $a=C_{n}$, then $A_{n}<B_{n}<C_{n}=a<b<c$. Therefore, setting $\beta$ := $b-A_{n}>0$ and $\gamma:=c-B_{n}>0$, we get

$$
(a, b, c) \stackrel{0, \beta, \gamma}{\longrightarrow}\left[C_{n}, A_{n}, B_{n}\right] .
$$

That is, we can apply rule $\mathbf{I}$ to reach the position $\left(A_{n}, B_{n}, C_{n}\right)$.

(b) If $a=B_{n}$, then we consider two sub-cases. 
(b.1) Assume $c \geq C_{n}$. In this case, since $A_{n}<B_{n}=a<b$, we set $\beta:=b-A_{n}>0$ and $\gamma:=c-C_{n}$. We get

$$
(a, b, c) \stackrel{0, \beta, \gamma}{\longrightarrow}\left[B_{n}, A_{n}, C_{n}\right]
$$

and we can therefore apply rule $\mathbf{I}$ to reach the position $\left(A_{n}, B_{n}, C_{n}\right) \in \mathcal{S}$.

(b.2) Otherwise $c<C_{n}$. Since we may assume that $a+b<c$, we have $2 B_{n}<c$ (because $\left.B_{n}=a<b\right)$. Consequently, $2 B_{n}<C_{n}$. On the other hand, $B_{n}-A_{n} \geq n$ (as show in the proof of Corollary 3.6, the difference between two consecutive elements in the set $\left\{B_{j}-A_{j} \mid j \geq 0\right\}$ is at least one). Thus, using Lemma 3.2, $B_{n} \geq A_{n}+n=C_{n}-B_{n}$ and we get a contradiction, $2 B_{n} \geq C_{n}$. So case (b.2) never occurs.

(c) Assume $a=A_{n}$. In this case, we consider three sub-cases.

(c.1) If $b \geq B_{n}$ and $c \geq C_{n}$, then $(a, b, c) \stackrel{\mathbf{I}}{\longrightarrow}\left(A_{n}, B_{n}, C_{n}\right)$.

(c.2) If $b<B_{n}$ and $c \geq C_{n}$. Set $t:=b-a>0$. In particular, $t<B_{n}-A_{n}$. Therefore there exists $0<m<n$ such that $t=B_{m}-A_{m}$ or $t=C_{m}-B_{m}$. Indeed, we know from Theorem 3.1 that $B_{j}-A_{j}=$ $\operatorname{Mex}\left\{B_{i}-A_{i}, C_{i}-B_{i} \mid 0 \leq i<j\right\}$. Moreover, $m>0$ because $t>0$.

(c.2.1) Assume $t=B_{m}-A_{m}$. Set $\alpha:=a-A_{m}>0$ and $\gamma:=c-C_{m}>0$. We notice that $b-B_{m}=a-A_{m}$ and we obtain

$$
(a, b, c) \stackrel{\alpha, \alpha, \gamma}{\longrightarrow}\left[A_{m}, B_{m}, C_{m}\right]
$$

If $\alpha \geq \gamma$ (resp. $\alpha<\gamma$ ) we conclude by applying rule II (resp. III).

(c.2.2) Assume $t=C_{m}-B_{m}$. Then $C_{m+1}-B_{m+1}=t+2$ or $t+3$. As a consequence of Corollary 3.6, there exists $K$ such that $B_{K}-A_{K}=t+1$. Notice that $B_{n}-A_{n} \geq t+1$ (because $t=b-a=b-A_{n}$ and $B_{n}>b$ ). So $K \leq n$. By Lemma 3.8, we know that

$$
t+1=C_{m}-B_{m}+1=B\left(A_{m}+1\right)-A\left(A_{m}+1\right)
$$

and we conclude that $K=A_{m}+1$. By Lemma 3.9, $B_{m}<A_{K}$. Since $K \leq n, A_{K} \leq A_{n}=a$ and we conclude that $B_{m}<a$. Since $b-a=t=$ $C_{m}-B_{m}$, we have that $C_{m}<b$. Finally, $A_{m}<C_{m}<C_{n} \leq c$. We set $\alpha:=a-B_{m}, \beta:=b-C_{m}, \gamma:=c-A_{m}$ and

$$
(a, b, c) \stackrel{\alpha, \beta, \gamma}{\longrightarrow}\left[B_{m}, C_{m}, A_{m}\right]
$$

Depending on the values $\alpha, \beta, \gamma>0$, the conclusion follows using rule II or III.

(c.3) Assume $b \geq B_{n}$ and $c<C_{n}$. Set $m:=c-a-b$, and notice by (6) that $m>0$. We show that

$$
(a, b, c) \stackrel{\text { II }}{\longrightarrow}\left(A_{m}, B_{m}, C_{m}\right) .
$$


First observe that $m \leq n-1$ because

$$
c-a-b \leq C_{n}-1-A_{n}-B_{n}=n-1
$$

where the last equality comes from Theorem 3.1. Next, notice that $c>C_{m}$. Indeed, we have

$$
c-C_{m}=m+a+b-\left(A_{m}+B_{m}+m\right)=A_{n}-A_{m}+b-B_{m}
$$

and since $n>m$, then $A_{n}>A_{m}$ and $b \geq B_{n}>B_{m}$. So up to now, we know that

$$
\alpha:=a-A_{m}>0, \beta:=b-B_{m}>0 \text { and } \gamma:=c-C_{m}>0
$$

but we still have to check that the conditions for applying II hold. First notice that $\max (\alpha, \beta, \gamma)=\gamma$. Indeed, it is clear that $A_{n}-A_{m} \leq B_{n}-B_{m}$ (the reader can refer to the proof of Corollary 3.6 for arguments) and since $b \geq B_{n}$, we conclude that $\alpha \leq \beta$. Moreover, it follows from the definitions that

$$
\begin{aligned}
\gamma-\beta & =c-\left(A_{m}+B_{m}+m\right)-\left(b-B_{m}\right) \\
& =c-b-A_{m}-(c-a-b)=a-A_{m}=A_{n}-A_{m}>0 .
\end{aligned}
$$

To conclude this part, we just have to show that $2 \gamma \leq \alpha+\beta+\gamma$. Here, inequality sign can in fact be replaced with an equality, because

$$
\alpha+\beta-\gamma=a+b-c-\left(A_{m}+B_{m}-C_{m}\right)=m-m=0 .
$$

(c.4) Assume $b<B_{n}$ and $c<C_{n}$. Once again, set $m:=c-a-b$.

(c.4.1) Assume as a first sub-case that $\alpha:=a-A_{m} \geq 0, \beta:=b-$ $B_{m} \geq 0$ and $\gamma:=c-C_{m} \geq 0$. If $\alpha \beta \gamma=0$ then one can immediately apply rule I. So let us assume now that $\alpha \beta \gamma>0$. Then $A_{n}=a>A_{m}$ and so $n>m$. Just as for (c.3), we would like to apply rule II. With the same justifications as in (c.3), one shows that $\beta<\gamma$. To obtain that $\max (\alpha, \beta, \gamma)=\gamma$, notice that

$$
\begin{aligned}
\gamma-\alpha & =c-\left(A_{m}+B_{m}+m\right)-a-A_{m} \\
& =c-a-B_{m}-(c-a-b)=b-B_{m}>0 .
\end{aligned}
$$

The conclusion of this case follows the same lines as (c.3), $\gamma=\alpha+\beta$.

(c.4.2) Assume that we are now in the situation where at least one of the elements $\alpha, \beta, \gamma$ defined in (c.4.1) is negative. Set

$$
M:=\max \left\{k \in \mathbb{N} \mid A_{k} \leq a, B_{k} \leq b, C_{k} \leq c\right\}
$$

Since $a=A_{n}$, we conclude that $M \leq n$. Moreover, $M \neq n$ since $b<B_{n}$ and $c<C_{n}$. So $M+1 \leq n$ and $A_{M+1} \leq A_{n}$ but by definition of $M$ we 
must therefore have $B_{M+1}>b$ or $C_{M+1}>c$. We consider these two cases separately.

(c.4.2.1) Suppose that $B_{M+1}>b$. Then $B_{M+1}-A_{M+1}>b-a$ (indeed remember that $\left.A_{M+1} \leq A_{n}=a\right)$. By Theorem 3.1, there exists $K \leq M$ such that $b-a=B_{K}-A_{K}$ or $C_{K}-B_{K}$. Moreover $b-a>0$ implies that $K>0$.

Assume first that $b-a=B_{K}-A_{K}$. Since $K \leq M<n$, for $X=A, B, C$ (resp. $x=a, b, c)$, we have $X_{K} \leq X_{M} \leq x$ and therefore $\alpha^{\prime}:=a-A_{K}=$ $b-B_{K}>0$ and $\gamma^{\prime}:=c-C_{K} \geq 0$, i.e.,

$$
(a, b, c) \stackrel{\alpha^{\prime}, \alpha^{\prime}, \gamma^{\prime}}{\longrightarrow}\left[A_{K}, B_{K}, C_{K}\right]
$$

Thus, if $\gamma^{\prime}=0$ we apply rule $\mathbf{I}$, otherwise if $\alpha^{\prime} \geq \gamma^{\prime}>0$ we apply rule II and if $\alpha^{\prime}<\gamma^{\prime}$ we apply rule III to obtain a position in $\mathcal{S}$.

Assume now that $b-a=C_{K}-B_{K}$. To conclude this part, we show that there exists $\alpha^{\prime}, \gamma^{\prime}>0$ such that

$$
(a, b, c) \stackrel{\alpha^{\prime}, \alpha^{\prime}, \gamma^{\prime}}{\longrightarrow}\left[B_{K}, C_{K}, A_{K}\right]
$$

Notice that $c \geq C_{K}>A_{K}$ so $\gamma^{\prime}:=c-A_{K}>0$. We develop now the same arguments as in case (c.2.2). By Lemma 3.8, we know that

$$
b-a+1=C_{K}-B_{K}+1=B\left(A_{K}+1\right)-A\left(A_{K}+1\right)
$$

Moreover $b-a<B_{n}-A_{n}$ and we conclude that $A_{K}+1 \leq n$ so $A\left(A_{K}+\right.$ $1) \leq A_{n}$. By Lemma 3.9, we have $B_{K}<A\left(A_{K}+1\right) \leq a$. Therefore $\alpha^{\prime}:=a-B_{k}=b-C_{k}>0$. Hence the conclusion follows using rule II or III depending as usual on the values $\alpha^{\prime}$ and $\gamma^{\prime}$.

(c.4.2.2) Suppose that $B_{M+1} \leq b$ and $C_{M+1}>c$. Since $A_{M+1} \leq a$, we have

$$
m-(M+1)=c-a-b-\left(C_{M+1}-A_{M+1}-B_{M+1}\right)<0
$$

and thus $m \leq M$ but this contradicts the fact that at least one of the elements $\alpha, \beta$ or $\gamma$ is negative. Consequently this case never occurs.

\section{Polynomial Winning StRategy}

We conclude this paper by discussing algorithmic complexity issues about the computation of the winning and losing positions of the Tribonacci game. Table 1 is the key argument to decide whether a given position is a $P$-position or not. For a given position $(a, b, c)$, one has to check whether it appears or not in the table. If one uses Theorem 3.1 to build Table 1 up to position $(a, *, *)$ then this process has an exponential complexity with respect to the size of the input $a$. Indeed, if an integer $a$ is represented in a given base $t$, whatever the base is or even in an exotic 
numeration system like the Tribonacci system, the length of the representation is $\left\lfloor\log _{t} a\right\rfloor+1$. So building the table up to $a$ has a linear complexity with respect to $a$, but an exponential complexity compared to $\left\lfloor\log _{t} a\right\rfloor$.

Another way to decide whether or not a given triple $(a, b, c)$ is a $P$-position would be to have an algebraic characterization of the sequences $\left(A_{n}\right)_{n \geq 0},\left(B_{n}\right)_{n \geq 0}$ and $\left(C_{n}\right)_{n \geq 0}$, as it is the case for Wythoff's game. For this latter game, $P$-positions $\left(a_{n}, b_{n}\right)$ are exactly $\left(\lfloor n \Phi\rfloor,\left\lfloor n \Phi^{2}\right\rfloor\right)$ where $\Phi$ is the golden ratio (see [19]). For the Tribonacci game, such a result does not exist as shown by the following theorem.

Theorem 5.1. There exist no real numbers $\alpha, \alpha^{\prime}, \beta, \beta^{\prime}, \gamma, \gamma^{\prime}$ such that $A_{n}=\lfloor n \alpha+$ $\left.\alpha^{\prime}\right\rfloor, B_{n}=\left\lfloor n \beta+\beta^{\prime}\right\rfloor$ or $C_{n}=\left\lfloor n \gamma+\gamma^{\prime}\right\rfloor$ for all $n \in \mathbb{N}$.

Proof. Let $\left(X_{n}\right)_{n \geq 1}$ be a nondecreasing sequence of integers. In [3], a necessary condition for the existence of real numbers $\delta, \delta^{\prime}$ such that $X_{n}=\left\lfloor n \delta+\delta^{\prime}\right\rfloor$ for all $n \geq 1$, is that for all $r \geq 2$,

$$
\underline{d}(r)=\max _{1 \leq i<k \leq r} \frac{X_{k}-X_{k-i}-1}{i}<\bar{d}(r)=\min _{1 \leq i<k \leq r} \frac{X_{k}-X_{k-i}+1}{i} .
$$

- By Theorem 2.4, we have $C_{k}-C_{k-1} \in\{4,6,7\}$. Hence applying the previous result to the sequence $\left(C_{n}\right)_{n \geq 1}$ and choosing in the above formula $i=1$ and $C_{k}-C_{k-1}=7$ (resp. 4$)$, we obtain $\underline{d}(r) \geq 6(\operatorname{resp} . \bar{d}(r) \leq 5)$. Consequently, we get $\bar{d}(r)<\underline{d}(r)$ and the conclusion follows for $\left(C_{n}\right)_{n \geq 0}$.

- Similarly, we have $B_{k}-B_{k-1} \in\{2,3,4\}$ and $\bar{d}(r) \leq 3 \leq \underline{d}(r)$.

- By considering the factor cabac (resp. abaaba) of $\mathbf{t}$ and in view of Remark 2.1, there exists $k$ (resp. $\ell$ ) such that $A_{k}-A_{k-5}=8$ (resp. $A_{\ell}-$ $\left.A_{\ell-6}=12\right)$. Therefore $\bar{d}(r) \leq 9 / 5<11 / 6 \leq \underline{d}(r)$ and the conclusion follows for $\left(A_{n}\right)_{n \geq 0}$.

Despite this negative result, the morphic formalism, and especially Remark 2.5, gives here a polynomial time algorithm to decide whether or not a given position is a $P$-position. This latter result is a direct consequence of the following statement combined with the proof of Theorem 4.1.

Theorem 5.2. A configuration $(a, b, c)$ is a P-position of the Tribonacci game if and only if there exists a word $w \in\{0,1\}^{*}$ such that $w$ does not contain three consecutive 1 's and $\rho_{T}(a-1)=w 0, \rho_{T}(b-1)=w 01$ and $\rho_{T}(c-1)=w 011$, where $\rho_{T}(n)$ is the Tribonacci representation of $n$.

Proof. We have already noticed in Remark 2.5 that $t_{n}=a\left(\right.$ resp. $\left.t_{n}=b, t_{n}=c\right)$ if and only if $\rho_{T}(n-1)$ ends with 0 (resp. 01, 11).

We also know that the $n$th occurrence of $c$ in $\mathbf{t}$ belongs to the image under $\tau$ of the $n$th occurrence of $b$ in $\mathbf{t}$ which appears itself in the image under $\tau$ of the $n$th occurrence of $a$. Recall the notation of Remark 2.5. Let $M_{i}$ be the $i$ th word in $M$. Assume $M_{i}$ leads to state $a$ in $\mathcal{A}$ (in particular $M_{i}$ is of the form $w 0$ for $i>1$ or $\left.M_{1}=\varepsilon\right)$. This means that $t_{i}=a$ and $\rho_{T}(i-1)=M_{i}$. Suppose $t_{i}$ is the $n$th occurrence of $a$ in $\mathbf{t}$. Since the $n$th occurrence of $b$ in $\mathbf{t}$ appears as the second letter 
in $\tau\left(t_{i}\right)$, by the properties of $\mathcal{A}$ (see [15]), $M_{i} 1=w 01$ produces this letter. With the same argument, $M_{i} 11=w 011$ produces the $n$th occurrence of $c$ in $\mathbf{t}$.

Example 5.3. The following table illustrates the previous result.

TABle 4. First $P$-positions and the corresponding Tribonacci representations.

\begin{tabular}{ccc|rrr}
\hline$A_{n}$ & $B_{n}$ & $C_{n}$ & $\rho_{T}\left(A_{n}-1\right)$ & $\rho_{T}\left(B_{n}-1\right)$ & $\rho_{T}\left(C_{n}-1\right)$ \\
\hline 1 & 2 & 4 & $\varepsilon$ & 1 & 11 \\
3 & 6 & 11 & 10 & 101 & 1011 \\
5 & 9 & 17 & 100 & 1001 & 10011 \\
7 & 13 & 24 & 110 & 1101 & 11011 \\
\hline
\end{tabular}

Deciding whether a given position $(a, b, c)$ is a $P$-position or not can be done in polynomial time as follows. One first computes $\rho_{T}(a-1), \rho_{T}(b-1), \rho_{T}(c-1)$. This can be done in $\mathcal{O}\left(\log _{2}(c)\right)$ because the length of $\rho_{T}(c-1)$ is proportional to $\log _{2}(c-1)$ and we apply a greedy algorithm to compute the coefficients of the Tribonacci representation. Then, it suffices to check (in linear time) the simple syntactic condition given by the previous theorem.

All this study around the Tribonacci game leads naturally to consider the following question.

Question 5.4. Could the Tribonacci game be generalized to a $k$-bonacci game with rules constructed in such a way that the losing positions are given by the positions of the nth occurrence of the letters $a_{1}, \ldots, a_{k}$ in the $k$-bonacci word given by the morphism $\xi\left(a_{i}\right)=a_{1} a_{i+1}$ for $i<k$ and $\xi\left(a_{k}\right)=a_{1}$ ?

According to our investigations, the answer might be positive but even for $k=4$ formulating the rules of such a game is tedious task.

Acknowledgements. We are indebted to the anonymous referees for their valuable advises and remarks to improve this paper.

\section{REFERENCES}

[1] E. Barcucci, L. Bélanger, S. Brlek, On Tribonacci sequences. Fibonacci Quart. 42 (2004) 314-319.

[2] E.R. Berlekamp, J.H. Conway, R.K. Guy, Winning ways (two volumes). Academic Press, London (1982).

[3] M. Boshernitzan, A. Fraenkel, Nonhomogeneous spectra of numbers. Discrete Math. 34 (1981) 325-327.

[4] L. Carlitz, R. Scoville, V.E. Hoggatt Jr., Fibonacci representations of higher order. Fibonacci Quart. 10 (1972) 43-69.

[5] A. Cobham, Uniform tag sequences. Math. Syst. Theor. 6 (1972) 164-192. 
[6] P. Fogg, Substitutions in dynamics, arithmetics and combinatorics, edited by V. Berthé, S. Ferenczi, C. Mauduit and A. Siegel. Lect. Notes Math. 1794, Springer-Verlag, Berlin (2002).

[7] A. Fraenkel, I. Borosh, A generalization of Wythoff's game. J. Combin. Theory Ser. A 15 (1973) 175-191.

[8] A. Fraenkel, How to beat your Wythoff games' opponent on three fronts. Amer. Math. Monthly 89 (1982) 353-361.

[9] A. Fraenkel, Systems of numeration. Amer. Math. Monthly 92 (1985) 105-114.

[10] A. Fraenkel, Heap games, numeration systems and sequences. Ann. Comb. 2 (1998) 197-210.

[11] A. Fraenkel, The Raleigh game, to appear in INTEGERS, Electron. J. Combin. Number Theor 7 (2007) A13.

[12] A. Fraenkel, The rat game and the mouse game, preprint.

[13] M. Lothaire, Combinatorics on words. Cambridge Mathematical Library, Cambridge University Press, Cambridge (1997).

[14] G. Rauzy, Nombres algébriques et substitutions. Bull. Soc. Math. France 110 (1982) $147-178$.

[15] M. Rigo and A. Maes, More on generalized automatic sequences. J. Autom. Lang. Comb. 7 (2002) 351-376.

[16] N.J.A. Sloane, On-Line Encyclopedia of Integer Sequences, see http://www.research.att.com/ njas/sequences/

[17] B. Tan, Z.-Y. Wen, Some properties of the Tribonacci sequence. Eur. J. Combin. 28 (2007) $1703-1719$.

[18] W.A. Webb, The length of the four-number game. Fibonacci Quart. 20 (1982) 33-35.

[19] W.A. Wythoff, A modification of the game of Nim. Nieuw Arch. Wisk. 7 (1907) 199-202.

[20] E. Zeckendorf, Représentation des nombres naturels par une somme des nombres de Fibonacci ou de nombres de Lucas. Bull. Soc. Roy. Sci. Liège 41 (1972) 179-182.

Communicated by J. Berstel.

Received May 7, 2007. Accepted September 26, 2007. 